\title{
Clinical effects of perazine ferulate tablets combined with eucalyptol limonene pinene enteric soft capsules for treatment of children with IgA nephropathy
}

\author{
ZHIZHEN LIU, JINGMEI PAN, CHUNLEI SUN, JUAN ZHOU and NA LI \\ Department of Pediatrics, Yidu Central Hospital of Weifang, Weifang, Shandong 262500, P.R. China
}

Received December 24, 2015; Accepted May 6, 2016

DOI: $10.3892 /$ etm.2016.3312

\begin{abstract}
The clinical effects of piperazine ferulate tablets combined with eucalyptol limonene pinene enteric soft capsules for treatment of children with IgA nephropathy were investigated. Sixty children with $\operatorname{Ig}$ A nephropathy were included in the study and were randomly divided into the control $(n=30)$ and observation $(n=30)$ groups. The patients in the control group were treated with conservative or hormone therapy while patients in the observation group were treated with piperazine ferulate tablets combined with eucalyptol-limonene-pinene enteric soft capsules. Clinical effects were observed and compared. The total effective rate of the observation group was significantly higher than that of the control group, while the incidence of complications was significantly lower than that of the control group $(\mathrm{p}<0.05)$. Serum IgA and fibronectin levels of the observation group were significantly lower than those of the control group, while the level of C3 was significantly higher than that of the control group $(\mathrm{p}<0.05)$. In conclusion, piperazine ferulate tablets combined with eucalyptus enteric soft capsule constituted a safe and effective for the treatment of children with IgA nephropathy. The treatment was superior to conservative or hormone therapy, and thus worthy of clinical promotion.
\end{abstract}

\section{Introduction}

The morbidity of $\operatorname{Ig}$ A nephropathy in children is $0.8 \%$. Most children have good prognosis, with $<5 \%$ of child patients showing rapidly progressive glomerulonephritis that develops into renal failure (1). At present, $\operatorname{IgA}$ nephropathy is caused by the precipitation of circulating immune complexes that contain IgA in kidney, and the antigen in the complex may be associated with the virus and bacteria from the respiratory

Correspondence to: Dr Zhizhen Liu, Department of Pediatrics, Yidu Central Hospital of Weifang, 4138 South Linglong Shan Road, Weifang, Shandong 262500, P.R. China

E-mail: vkg778@163.com

Key words: piperazine ferulate tablets, eucalyptol-limonene-pinene enteric soft capsule, IgA nephropathy tract or gastrointestinal tract mucosa or certain components of food (2). The typical symptoms include hematuria (75\%), albuminuria $(73 \%)$, low fever $(65 \%)$, kidney area percussion pain $(65 \%)$, small vascular fibrous necrosis $(55 \%)$, and edema (55\%) (3). Treatment suggestions include good rest, anti-infection and symptomatic treatment, if necessary, combined with adrenal cortical hormone and immunosuppressive therapy (4).

In Chinese medicine, the processing, extraction, concentration and blending of certain components of some plants, produces piperazine ferulate tablets and eucalyptol-linonene-pinene enteric soft capsules. Previous findings confirmed that the two drugs can inhibit IgA from depositing in the kidney and can reduce the immune response (5).

In the present study, the safety and efficacy of a combination of the two drugs for the treatment of children with IgA nephropathy were also evaluated and clinical effects were compared with conventional western medicine to provide new ideas for clinical treatment.

\section{Patients and methods}

Patients. Sixty children with IgA nephropathy were included in the study. Diagnostic criteria of IgA nephropathy included: i) Clinical symptoms such as gross hematuria, and proteinuria; ii) kidney tissue puncture immune pathological examinations: a) light microscope showed glomerular number $>6$; b) immune fluorescence showed characteristic changes, immunoglobulin dominated by IgA showed granular or lump-like diffuse deposits in glomerular mesangial region, and some deposits alongside capillary loop; and c) electron microscope showed mesangial cell proliferation, increased mesangial matrix with large lump-like electron dense deposits; and iii) excluded Henoch-Schönlein Purpura nephritis, lupus nephritis, sicca syndrome, psoriasis, ankylosing spondylitis, liver cirrhosis, hepatitis $\mathrm{B}$ or $\mathrm{C}$ virus infection and other secondary $\operatorname{Ig} \mathrm{A}$ nephropathy. Patients that conformed to the diagnostic criteria of IgA nephropathy and patients that were treated for the first time were also included in the study.

Exclusion criteria were, patients with nephrotic syndrome, acute nephritis, acute nephritis and renal failure; patients with uncontrolled infection, fever and diarrhea; patients with congenital malformation, hereditary metabolic disease, combined with other organ dysfunction and coagulation disorders; patients with allergic or intolerable to piperazine ferulate 
Table I. Comparison of total efficiency and the incidence of complications [case (\%)].

\begin{tabular}{|c|c|c|c|c|c|c|c|c|c|}
\hline Group & Case & Excellent $^{\mathrm{a}}$ & Effective $^{b}$ & Ineffective $^{c}$ & $\begin{array}{l}\text { Total } \\
\text { effective } \\
\text { rate }\end{array}$ & $\begin{array}{l}\text { Secondary } \\
\text { infection }\end{array}$ & $\begin{array}{c}\text { Renal } \\
\text { function } \\
\text { deterioration }\end{array}$ & $\begin{array}{l}\text { Stress } \\
\text { ulcer }\end{array}$ & Complication \\
\hline Control & 30 & 9 & 10 & 11 & $19(63.3)$ & 3 & 5 & 2 & $10(33.3)$ \\
\hline Observation & 30 & 13 & 13 & 4 & $26(86.7)$ & 1 & 2 & 0 & $3(10.0)$ \\
\hline$\chi^{2}$ test & & & & & 4.356 & & & & 4.812 \\
\hline P-value & & & & & 0.037 & & & & 0.028 \\
\hline
\end{tabular}

${ }^{\mathrm{a} E x c e l l e n t, ~ c l i n i c a l ~ s y m p t o m s ~ d i s a p p e a r e d ~ a n d ~ I g A ~ d e p o s i t i o n ~ d e c r e a s e d ~ b y ~}>90 \%$. ${ }^{\mathrm{b}}$ Effective, clinical symptoms mostly disappeared and IgA deposition decreased by 50-80\%. 'Ineffective, clinical symptoms were not improved. ${ }^{\mathrm{d} C o m p l i c a t i o n s ~ i n c l u d e d ~ I g A ~ s e c o n d a r y ~ h y p e r t e n s i o n, ~}$ increased proteinuria, secondary infection, renal function deterioration, and stress ulcer.

tablets and eucalyptol-linonene-pinene enteric soft capsule, with poor compliance.

After obtaining the approval of the Ethics Committee of the Yidu Central Hospital of Weifang (Shandong, China) and the informed consent of the parents or relatives, the patients were divided randomly into the control $(\mathrm{n}=30)$ and observation $(n=30)$ group. The control group included 19 male and 11 female patients, with an age range of 6-15 years, and an average of $12.3 \pm 4.2$ years. The disease course ranged from 3 days to 2 months, and an average of $27.4 \pm 8.2$ days. The observation group comprised 20 male and 10 female patients, with an age range of 5-17 years and an average age of $13.6 \pm 5.5$ years. The disease course ranged from 5 days to 2 months and an average of $26.3 \pm 7.7$ days. Differences in gender, age and course of the two groups were not statistically significant $(\mathrm{p}>0.05)$.

Treatment methods. Patients in the control group were treated with conservative or hormone therapy. Conservative therapy included good rest, antiviral or antibiotic anti-infection, full course of treatment, and symptomatic treatments such as diet control, moderate depressurization, and protein reduction. The patients that failed conservative treatment used hormone or hormone combined with immunosuppressive therapy. Dosage regimen included the oral administration of prednisone, initial dose of $1 \mathrm{mg} / \mathrm{kg} . \mathrm{d}$, taken in the morning, maintained for 8 weeks and then gradually reduced the dose by $5 \mathrm{mg}$ every week until $5 \mathrm{mg} /$ day, followed by maintaining the dose for a total course of 6 months. During the treatment period, any complications were closely monitored and the dose or suspended medication were adjusted in a timely manner if necessary.

Patients in the observation group were treated using piperazine ferulate tablets combined with eucalyptol-limonene-pinene enteric soft capsules. Dosage regimen included $0.1 \mathrm{~g}$ piperazine ferulate tablets $(50 \mathrm{mg} /$ tablet; Chengdu Heand Pharmeuticals Co., Ltd., Chengdu, China), 3 times/day; and eucalyptol-limonene-pinene enteric soft capsule (KPC Pharmaceuticals, Inc., Kunming, China), 2 capsules/time, twice per day for 6 months. During the treatment period, the patients were closely monitored for any complications and dose or suspended medication was adjusted in a timely manner if necessary.
Observation indices. The differences of total efficiency, incidence of complication, serum IgA, fibronectin and complement C3 levels of the two groups were compared (Table I).

Detection of serum IgA, fibronectin and complement $C 3$. Venous blood (5 $\mathrm{ml})$ was drawn in the morning and centrifuged at 2,000 $\mathrm{x}$ g for $15 \mathrm{~min}$ to collect the supernatant for preservation at $-80^{\circ} \mathrm{C}$. Kits of $\operatorname{IgA}$, fibronectin and complement $\mathrm{C} 3$ were purchased from Santa Cruz Biotechnology, Inc. (Santa Cruz, CA, USA). IgA was detected using ELISA and fibronectin and complement $\mathrm{C} 3$ were examined by single radial immunodiffusion, according to the supplier instructions.

Statistical analysis. SPSS 19.0 statistical software (SPSS Inc, Chicago, IL, USA) was used for statistical analysis and data were presented as mean \pm standard deviation. The comparison between groups was made by an independent sample t-test. Enumeration data were expressed as a percentage. The comparison between groups was made using the $\chi^{2}$ test. $P<0.05$ was considered to indicate a statistically significant difference.

\section{Results}

Comparison of total efficiency and the incidence of complications. Twelve patients in the control group underwent expectant treatment and 18 cases underwent hormonal treatment. The total effective rate of the observation group was significantly higher compared to the control group. The incidence of complications was significantly lower compared to the control group $(\mathrm{p}<0.05)$.

Comparison of serum IgA, fibronectin and complement $C 3$ levels. Prior to intervention, differences of serum IgA, fibronectin and complement $\mathrm{C} 3$ levels of the two groups were not statistically significant $(\mathrm{p}>0.05)$. After intervention, the indices of the two groups were improved and serum IgA and fibronectin levels of the observation group were significantly lower than those of the control group. Levels of complement $\mathrm{C} 3$ were significantly higher than that of the control group $(\mathrm{p}<0.05)$ (Table II).

\section{Discussion}

$\operatorname{Ig} \mathrm{A}$ nephropathy is accompanied by deposition of $\operatorname{Ig} \mathrm{A}$ and $\mathrm{C} 3$ deposition in kidney tissues. Ig A is an important 
Table II. Comparison of serum IgA, fibronectin and complement C3 levels.

\begin{tabular}{|c|c|c|c|c|c|c|}
\hline \multirow[b]{2}{*}{ Group } & \multicolumn{2}{|c|}{$\operatorname{IgA}$} & \multicolumn{2}{|c|}{ Fibronectin $(\mu \mathrm{g} / \mathrm{ml})$} & \multicolumn{2}{|c|}{ Complement C3 (g/l) } \\
\hline & Pre-intervention & Post-intervention & Pre-intervention & Post-intervention & Pre-intervention & Post-intervention \\
\hline Control & $0.82 \pm 0.05$ & $0.59 \pm 0.04$ & $430.6 \pm 52.3$ & $328.7 \pm 38.9$ & $0.5 \pm 0.2$ & $0.7 \pm 0.3$ \\
\hline Observation & $0.84 \pm 0.06$ & $0.33 \pm 0.02$ & $442.1 \pm 46.7$ & $265.4 \pm 30.2$ & $0.4 \pm 0.1$ & $1.1 \pm 0.3$ \\
\hline t-test & 0.624 & 5.032 & 0.327 & 5.127 & 0.935 & 5.263 \\
\hline P-value & 0.573 & 0.025 & 0.128 & 0.021 & 0.763 & 0.017 \\
\hline
\end{tabular}

immunoglobulin that accounts for $15 \%$ of the total serum immunoglobulin (6). IgA deposition is parallel to pathological changes of glomerulus. $\operatorname{Ig} \mathrm{A}$ deposition in the mesangial area is accompanied by mesangial proliferation, and $\operatorname{IgA}$ deposition in the capillary is accompanied by the changes of blood vessel endothelium (7). Pathological factors that cause IgA deposition include $(3,4,7)$ : i) Entrance of an antigen into the body from mucosa to stimulate the $\operatorname{IgA}$ immune system. Antigen may be a microorganism or food such as egg albumin, bovine serum albumin, and casein; ii) abnormal IgA immune response results in the formation of poly IgA of high molecular weight; iii) poly $\operatorname{IgA}$ that contains antigens combines with fibronectin through static $\lambda$ chain or receptor FeaR and precipitates in kidney. IgA-fibronectin complex in serum was characteristic of IgA nephropathy; and iv) other IgA removal mechanisms such as liver damage or saturation.

Previous findings showed that $\operatorname{IgA}$ that precipitate in glomerulus of $\operatorname{Ig}$ A nephropathy were mainly poly $\operatorname{Ig} \mathrm{A}$ (8). $\operatorname{Ig} \mathrm{A}$ nephropathy patients showed increased serum IgA1, poly IgA, and $\lambda$-IgA1. The lymphoid $B$ cells had $\beta-1$ and 33 galactose transferase deficiency, which led to O-type glycosylation of the IgA1 strand area and reduced galactolipin of chain end, which further affected the binding of $\operatorname{IgA} 1$ with oligo sialic acid protein receptor ASGPR in liver cells. The affected $\operatorname{Ig}$ A removal increased the binding of $\operatorname{Ig} \mathrm{A}$ with other kidney tissues, leading to its deposition (9).

$\operatorname{Ig}$ A nephropathy in western countries is generally treated by hormone or immunosuppressive therapy, which although effective, is prone to recurrence, hormone dependence and related complications, and other side effects (3). In a related study(4) it was identified that, for child patients, conservative treatment was able to receive the same curative effects as hormone or hormone combined with immunosuppressive agents, and with fewer complications.

Eucalyptol-limonene-pinene enteric soft capsule has been developed from the ferulaic acid of ligustrazine, a traditional Chinese medicine. Basic studies (10) have confirmed that it may promote circulation and remove stasis, inhibit platelet aggregation, activate meridians and collaterals, and exerts anti-inflammatory and anti-oxidative effects. Its possible mechanisms involve selectively releasing thromboxane synthase, inhibiting the activation of phospholipase A2, reducing the generation of peanut arachidonic acid, improving the level of cyclic AMP, inhibiting activity of phosphodiesterase, inhibiting the expression of growth factors of glomerular mesangial cell connective tissues and synthesis of extracellular matrix, inhibiting the activation of renal fibroblast phenotype, reducing the abnormal expression of $\operatorname{Ig} \mathrm{A}$ in circulation or in situ, and inhibiting antigen-antibody complex from precipitating in renal mesangial region (11). Another study (12) also suggested that piperazine ferulate was effective in nephrotic syndrome and diabetic nephropathy. It may reduce the pressure inside the glomerulus, prevent glomerular sclerosis, inhibit platelet aggregation, anti-coagulation, improve micro-circulation, inhibit cholesterol synthesis, reduce blood lipids, eliminate free radicals and prevent lipid peroxidation injury through the blood vessels constriction and vascular smooth muscle proliferation induced by antagonistic vascular endothelium.

Eucalyptol-limonene-pinene enteric soft capsule is composed of the extracts of Myrtaceae eucalyptus genus, Rutaceae citrus genus and Pinaceae pine genus plants (13). Its main components are eucalyptus, limonene and $\alpha$-pinene. Eucalyptol-limonene-pinene enteric soft capsule has many effects, such as promoting circulation and removing stasis, inhibiting platelet aggregation, activating meridians and collaterals, and exerting anti-inflammatory and antioxidative effects (14). Previous studies have found that the eucalyptol-limonene-pinene enteric soft capsule, as a natural and strongly effective mucus promoting agent, and may significantly improve the respiratory diseases especially symptoms associated with acute exacerbation of chronic bronchitis (15). Clinical application has proven that it can reduce the high reactivity of the airway, enhance the body immunity, and reduce the recurrence of respiratory tract infections (16).

The results of the present study have shown that the total effective rate, incidence of complications, serum $\operatorname{IgA}$ and fibronectin levels, and levels of complement C3 of the observation group were significantly higher compared to the control group. In conclusion, piperazine ferulate tablets combined with eucalyptol-limonene-pinene enteric soft capsule is safe and effective for the treatment of children with $\operatorname{IgA}$ nephropathy. It was superior to conservative or hormonal therapy, and thus worthy of clinical promotion.

\section{References}

1. Hastings MC, Moldoveanu Z, Suzuki H, Berthoux F, Julian BA, Sanders JT, Renfrow MB, Novak J and Wyatt RJ: Biomarkers in IgA nephropathy: relationship to pathogenetic hits. Expert Opin Med Diagn 7: 615-627, 2013.

2. Aizawa M, Suzuki Y, Suzuki H, Pang H, Kihara M, Nakata J, Yamaji K, Horikoshi S and Tomino Y: Uncoupling of glomerular $\operatorname{IgA}$ deposition and disease progression in alymphoplasia mice with IgA nephropathy. PLoS One 9: e95365, 2014.

3. Suzuki Y, Suzuki H, Nakata J, Sato D, Kajiyama T, Watanabe T and Tomino Y: Pathological role of tonsillar B cells in Ig A nephropathy. Clin Dev Immunol 2011: 639074, 2011. 
4. Kiryluk K and Novak J: The genetics and immunobiology of IgA nephropathy. J Clin Invest 124: 2325-2332, 2014.

5. Liu SJ, Gu Y, Li SJ, et al: Nephroprotective effects of piperazine ferulate on rat remnant kidney. Chin J Integr Trad West Nephrol 3: 256-259, 2002.

6. Yuzawa Y, Yamamoto R, Takahashi K, Katafuchi R, Tomita M, Fujigaki Y, Kitamura H, Goto M, Yasuda T, Sato M, Urushihara M, Kondo S, Kagami S, Yasuda Y, Komatsu H, Takahara M, Harabuchi Y, Kimura K, Matsuo S: Evidence-based clinical practice guidelines for IgA nephropathy 2014. Clin Exp Nephrol: April 21st, 2016 (Epub ahead of print).

7. Kalantari S, Rutishauser D, Samavat S, Nafar M, Mahmudieh L, Rezaei-Tavirani $M$ and Zubarev RA: Urinary prognostic biomarkers and classification of IgA nephropathy by high resolution mass spectrometry coupled with liquid chromatography. PLoS One 8: e80830, 2013.

8. Lai KN, Chan LY, Tang SC, Tsang AW, Guo H, Tse KC, Yip T and Leung JC: Characteristics of polymeric lambda-IgA binding to leukocytes in IgA nephropathy. J Am Soc Nephrol 13: 2309-2319, 2002.

9. Leung JC, Tsang AW, Chan DT and Lai KN: Absence of CD89, polymeric immunoglobulin receptor, and asialoglycoprotein receptor on human mesangial cells. J Am Soc Nephrol 11: 241-249, 2000

10. Zhang J, Chen J, Yang J, Xu C, Ding J, Yang J, Guo Q, Hu Q and Jiang H: Sodium ferulate inhibits neointimal hyperplasia in rat balloon injury model. PLoS One 9: e87561, 2014.
11. Wang W, Pei X, Xu M, Sun S, Zhang C, Mu K and Liu Z: The protective effect of sodium ferulate and oxymatrine combination on paraquat-induced lung injury. Iran J Pharm Res 14: 573-583, 2015.

12. Qin J, Shang L, Ping AS, Li J, Li XJ, Yu H, Magdalou J, Chen LB and Wang $\mathrm{H}$ : TNF/TNFR signal transduction pathway-mediated anti-apoptosis and anti-inflammatory effects of sodium ferulate on IL-1 $\beta$-induced rat osteoarthritis chondrocytes in vitro. Arthritis Res Ther 14: R242, 2012.

13. Qiu H, Fan W, Fu P, Zuo C, Feng P, Liu F, Zhou L, Chen F, Zhong H, Liang Y and Shi M: General acteoside of Rehmanniae leaves in the treatment of primary chronic glomerulonephritis: A randomized controlled trial. Afr J Tradit Complement Altern Med 10: 109-115, 2013.

14. Tyagi AK, Bukvicki D, Gottardi D, Tabanelli G, Montanari C, Malik A and Guerzoni ME: Eucalyptus essential oil as a natural food preservative: in vivo and in vitro antiyeast potential. Biomed Res Int 2014: 969143, 2014.

15. Dhibi S, Mbarki S, Elfeki A and Hfaiedh N: Eucalyptus globulus extract protects upon acetaminophen-induced kidney damages in male rat. Bosn J Basic Med Sci 14: 99-104, 2014.

16. Bokaeian M, Nakhaee A, Moodi B and Ali Khazaei H: Eucalyptus globulus (eucalyptus) treatment of candidiasis in normal and diabetic rats. Iran Biomed J 14: 121-126, 2010. 